Notes \& Tips

\title{
A streamlined protocol for emulsion polymerase chain reaction and subsequent purification
}

\author{
Tatjana Schütze ${ }^{\mathrm{a}, \mathrm{b}}$, Florian Rubelt ${ }^{\mathrm{a}, \mathrm{b}}$, Julia Repkow ${ }^{\mathrm{a}}$, Nicole Greiner ${ }^{\mathrm{a}}$, Volker A. Erdmann ${ }^{\mathrm{b}}$, Hans Lehrach ${ }^{\mathrm{a}}$, \\ Zoltán Konthur a , Jörn Glökler ${ }^{\mathrm{a}, *}$ \\ a Department of Vertebrate Genomics, Max Planck Institute for Molecular Genetics, Ihnestrasse 63-73, 14195 Berlin, Germany \\ ${ }^{\mathrm{b}}$ Institute for Chemistry/Biochemistry, Freie Universität Berlin, Thielallee 63, 14195 Berlin, Germany
}

\section{A R T I C L E I N F O}

Article history:

Received 21 September 2010

Received in revised form 12 November 2010

Accepted 17 November 2010

Available online 25 November 2010

\begin{abstract}
A B S T R A C T
Compartmentalization of polymerase chain reaction (PCR) reduces artifacts, especially when complex libraries are amplified. It allows clonal amplification of templates from complex mixtures in a bias-free manner. Here we describe a rapid, straightforward, and easy protocol for PCR in a water-in-oil emulsion (ePCR) including sample recovery by DNA purification. Furthermore, no special laboratory equipment is needed and inexpensive components are used. Therefore, our flexible protocol allows ePCR to be readily implemented in daily routine experiments for a broad range of applications.
\end{abstract}

(c) 2010 Elsevier Inc. All rights reserved.
The usefulness of applying emulsion PCR (ePCR $)^{1}$ is clearly demonstrated by its routine application in next-generation sequencing techniques. Amplification of DNA samples with a high diversity, such as genomic or cDNA libraries, or other complex mixtures of different sequences by conventional PCR may be compromised due to three general problems. Artifacts are generated by recombination between homologous regions, which results in the formation of chimeric DNA molecules [1]. Additionally, short fragments tend to be amplified more efficiently in comparison to longer ones [2]. Also, highly diverse nucleic acids may not be able to form perfect duplexes following denaturation steps in PCR [3].

A water-in-oil emulsion allows many reactions to occur independently in a single reaction tube. Distinct reaction compartments such as micelles provide controlled conditions by simple physical separation. Thereby each droplet can be regarded as equivalent to a separate test tube.

The advantages of ePCR are inhibition of trans-priming of different fragments, no biased amplification due to unequal amplification rates, and the same conditions for all individual sequences/ DNA templates are applied.

Current applications are ePCR on microbeads used for next-generation sequencing, such as the SOLID or 454 technology $[2,4]$, diagnostic DNA amplifications like BEAMing [5], or selections by in vitro compartmentalization [6]. Additionally, ePCR can be employed for the bias-free amplification of DNA libraries, e.g., cDNAs

\footnotetext{
* Corresponding author. Fax: +49 (0) 3084131380

E-mail address: gloekler@molgen.mpg.de (J. Glökler).

${ }^{1}$ Abbreviations used: BSA, bovine serum albumin; ePCR, emulsion PCR; PCR, polymerase chain reaction.
}

and other artificial highly diverse nucleic acids used in SELEX experiments. For many of these applications, the anchoring to beads in emulsions is not required. In such cases, the emulsions are usually broken by the addition of the highly volatile and flammable organic solvent diethyl ether followed by a precipitation step to isolate the amplified DNA [2,7]. In order to simplify the ePCR protocol (Fig. 1), we replaced the use of a sonifier or magnetic stirrer for compartmentalization by vortexing and simplified the DNA purification process by omitting precipitation. Instead, we adapted the procedure to be compatible with commercially available DNA cleanup kits.

We could determine that a homogeneous emulsion comparable to that of Diehl et al. [5] is readily formed by vigorous vortexing after 3 min (Supplementary Fig. S2). To show that our simplified ePCR protocol works efficiently, we have first applied it to the initial amplification of a cDNA library directly after reverse transcription. We demonstrated that the size distribution of DNA is similar to that in an open PCR (nonemulsified control); however, no shifts occur (Fig. 2a). Further, we show that ePCR enhances the quality of amplification of a diversified nucleic acid SELEX library by reducing artifact formation (Fig. 2b). We demonstrate that the protocol reported here preserves the diversity and complexity in DNA libraries in accordance with other ePCR amplification procedures.

PCR mixtures in 50- $\mu$ l aliquots (aqueous phase) were prepared on ice under the same conditions as open PCR, containing $2.5 \mu \mathrm{l}$ BSA $(10 \mathrm{mg} / \mathrm{ml}, \mathrm{NEB}, \mathrm{USA})$. As BSA content may depend on which PCR kit is used, it is advisable to verify the required amount of BSA by titration. Since the distribution of template DNA in micelles is in concordance with Poisson distribution, the total number of DNA molecules should not exceed $10^{9}$ copies. This ensures the 


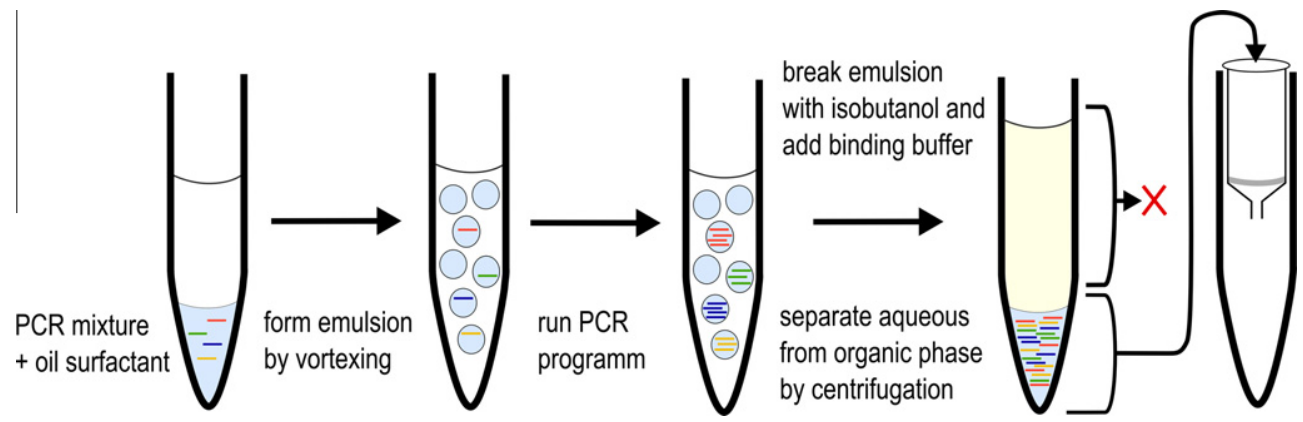

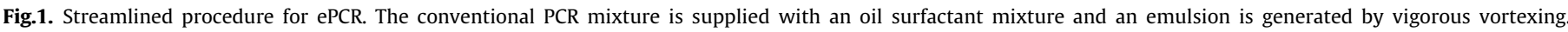

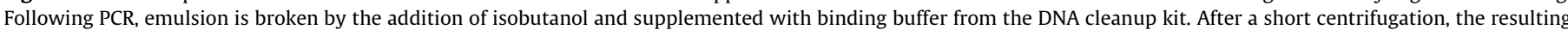
organic phase is removed and the remaining aqueous phase is subjected to DNA purification according to the kit protocol.
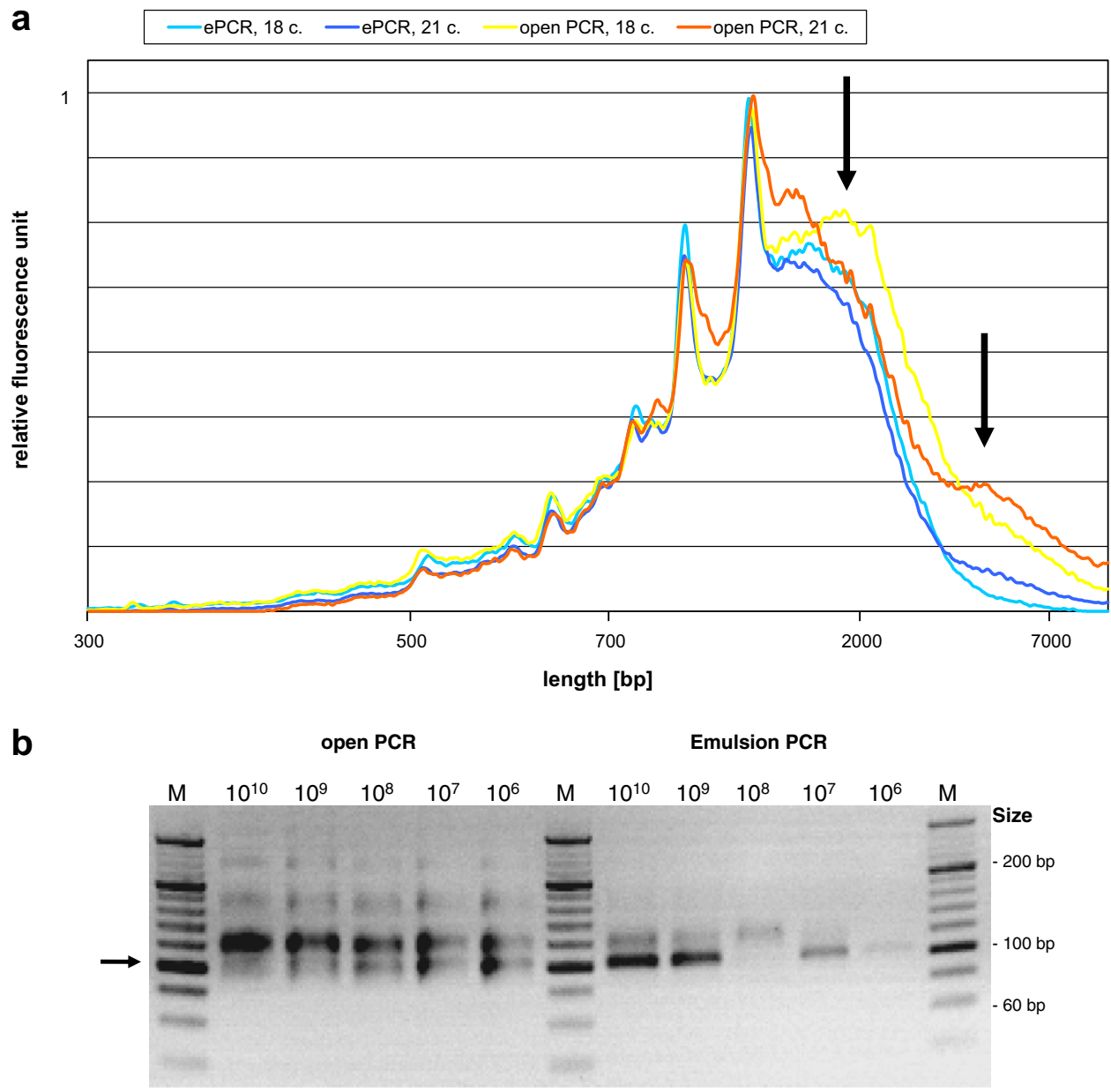

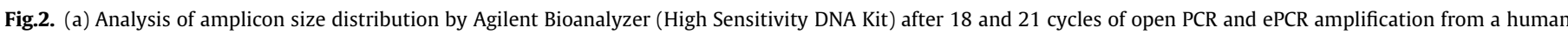

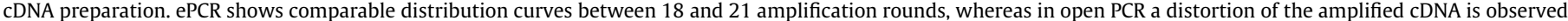

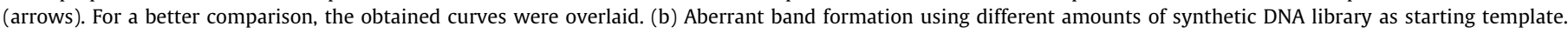
Arrow indicates correct template size. In contrast to ePCR, the open PCR produces significantly more artifacts as visualized by ethidium bromide on a $3 \%$ agarose gel.

presence of one or merely a few templates per micelle, a prerequisite for an unbiased amplification (Fig. 2b) $[2,8]$. The components for the emulsion were combined according to Diehl et al. [5]. Briefly, $300 \mu \mathrm{l}$ of prechilled oil surfactant containing $73 \%$ Tegosoft (Evonik, Germany), 20\% mineral oil (Sigma, Germany), and 7\% ABIL WE (Evonik) was added to the $50-\mu$ l aqueous phase. Water-in-oilemulsions were generated by putting the samples on a standard Vortex-Genie 2 vortexer (Scientific Industries Inc., USA) at full speed $(2700 \mathrm{rpm})$ for $5 \mathrm{~min}$ at $+10^{\circ} \mathrm{C}$ in a cold room. The resulting emulsion was divided into $100-\mu$ aliquots in PCR cups and incubated for 15-20 PCR cycles in a thermal cycler. Denaturation temperature did not exceed $95^{\circ} \mathrm{C}$.

To break the emulsion, corresponding samples were pooled in a 2 - $\mathrm{ml}$ reaction cup and $1 \mathrm{ml}$ isobutanol was added followed by short vortexing until the sample clarifies (usually $\sim 5 \mathrm{~s}$ ). From several tested organic solvents, isobutanol proved optimal for handling and efficiency (see Supplementary material). Following addition of DNA binding buffer from the DNA cleanup kit and 
subsequent vortexing, the organic and aqueous phases were easily separated by centrifugation for $1 \mathrm{~min}$ at $16,000 \mathrm{~g}$. The organic phase was discarded and DNA was purified with a DNA cleanup kit from the remaining aqueous phase. Multiple DNA cleanup kits were validated and best results were obtained using the GeneMatrix PCR/ DNA kit from EURx (Poland; purchased from Roboklon, Germany). Amplified DNA in the aqueous phase could also be purified with magnetic beads using the Sbeadex magnetic beads from LGC Genomics (England) according to the manufacturers directions (data not shown).

In order to monitor the correct amplification of DNA and to verify whether the numbers of amplification cycles are sufficient, it is advisable to use a small aliquot of an open PCR sample that was run in parallel reaction as a control in agarose gel electrophoresis. If the thermal stability of the emulsion during PCR needs to be checked, we recommend emulsifying primers and template separately, and pooling the generated emulsions in a single PCR tube. Stability should result in the absence of PCR product in comparison to the normal control setup.

The described protocol is compatible with modern high-throughput techniques. By using commercially available components for purification, it can be easily combined with existing automated processes. Next to the described examples of ePCR with cDNA and SELEX libraries (Fig. 2), we envisage that our ePCR protocol is applicable in many research areas and is not restricted to mere DNA amplification (PCR). It is possible to integrate ePCR into other protocols related to handling of diverse libraries such as gDNA and cDNA amplification, haplotyping, directed molecular evolution, and selection processes, e.g., for optimizing properties of novel enzymes and more [9].

\section{Acknowledgments}

This work was partially supported by the European Union through the EFRE Program (ProFIT Grant, Contract No. 10139409) and by the German Ministry of Science and Education (BMBF, Grant 0315448B).

\section{Appendix A. Supplementary data}

Supplementary data associated with this article can be found, in the online version, at doi:10.1016/j.ab.2010.11.029.

\section{References}

[1] A. Meyerhans, J. Vartanian, S. Wain-Hobson, DNA recombination during PCR, Nucleic Acids Res. 18 (1990) 1687-1691.

[2] R. Williams, S.G. Peisajovich, O.J. Miller, S. Magdassi, D.S. Tawfik, A.D. Griffiths, Amplification of complex gene libraries by emulsion PCR, Nat. Methods 3 (2006) 545-550.

[3] T. Schütze, M. Menger, A. Wochner, M. Vingron, V.A. Erdmann, H. Lehrach, et al., A calibrated diversity assay for nucleic acid libraries using DiStRO - a diversity standard of random oligonucleotides, Nucleic Acids Res. 38 (2010) e23.

[4] Y. Schaerli, F. Hollfelder, The potential of microfluidic water-in-oil droplets in experimental biology, Mol. BioSyst. 5 (2009) 1392.

[5] F. Diehl, M. Li, Y. He, K.W. Kinzler, B. Vogelstein, D. Dressman, BEAMing: singlemolecule PCR on microparticles in water-in-oil emulsions, Nat. Methods 3 (2006) 551-559.

[6] D.S. Tawfik, A.D. Griffiths, Man-made cell-like compartments for molecular evolution, Nat. Biotechnol. 16 (1998) 652-656.

[7] Q. Ge, Z. Liu, Y. Bai, D. Zhang, P. Yu, Z. Lu, Emulsion PCR-based method to detect Y chromosome microdeletions, Anal. Biochem. 367 (2007) 173-178.

[8] A. Swami, G. Espinosa, S. Guillot, E. Raspaud, F. Boué, D. Langevin, Confinement of DNA in water-in-oil microemulsions, Langmuir 24 (2008) 11828-11833.

[9] A.D. Griffiths, D.S. Tawfik, Miniaturising the laboratory in emulsion droplets, Trends Biotechnol. 24 (2006) 395-402. 